\title{
Insulin secretion and impaired glucose tolerance
}

\author{
A. B. Jenkins • L. V. Campbell
}

Received: 17 April 2010 /Accepted: 30 April 2010 /Published online: 22 May 2010

(C) Springer-Verlag 2010

To the Editor: The recent publication in Diabetologia of baseline results from two large-scale longitudinal studies provides welcome confirmation of the association of impaired beta cell function and glucose intolerance outside the diabetic range and promises useful information when follow-up studies are completed regarding factors related to progression of glucose intolerance [1, 2]. While both studies conclude that beta cell function is the dominant determinant of glucose tolerance, they differ in one significant detail. Mari et al. [1] conclude that variations in their primary index of insulin secretion (glucose sensitivity) are not associated with variations in wholebody insulin sensitivity and therefore are not related to any feedback relationship between beta cell function and insulin sensitivity. On the contrary, DeFronzo et al. [2] conclude from their primary index of insulin secretion (a disposition index [DI]), that impairments do represent a failure of feedback between insulin sensitivity and insulin secretion. We present here an argument that the two different interpretations reflect the same underlying reality, which is closest to the interpretation of Mari et al., and that the apparent difference is due to a misinterpretation of the physiological and statistical relationships between insulin secretion, insulin sensitivity and glucose tolerance (or other measures of glycaemia).

A. B. Jenkins $(\bowtie)$

School of Health Sciences, University of Wollongong,

Northfields Avenue,

Wollongong, NSW, Australia 2522

e-mail: ajenkins@uow.edu.au

A. B. Jenkins $\cdot$ L. V. Campbell

Garvan Institute of Medical Research,

Darlinghurst, NSW, Australia

L. V. Campbell

St Vincent's Hospital,

Darlinghurst, NSW, Australia
We have shown elsewhere [3], using published data from some of the authors of Mari et al. and DeFronzo et al. [4], that glycaemic responses to an OGTT conform closely to a simple HOMA-like model [5] relating insulin secretion, insulin sensitivity and glycaemia. The model can be expressed as:

$\ln (\beta)=\ln \left(R_{\text {in }}\right)-\ln \left(S_{\mathrm{i}}\right)-\ln (G)$

where $\beta$ is the glucose sensitivity of insulin secretion, $R_{\text {in }}$ is the average rate of entry of glucose assumed to be invariant between individuals, $S_{\mathrm{i}}$ is whole-body insulin sensitivity and $G$ is a measure of glycaemia. It is important to note that this model cannot account for the dynamics of glucose in response to OGTT or other stimuli, but only for responses obtained at steady state (or a reasonable approximation to it, such as during fasting or $2 \mathrm{~h}$ into an OGTT), or for integrated responses such as the area under a glycaemic response curve during OGTT or IVGTT.

That this model provides a strong fit to data $\left(r^{2}=0.83\right.$ for both fasting and $2 \mathrm{~h}$ OGTT responses [4]) is not proof that it is fundamentally true or complete. However, it demonstrates that, in this data set at least, the model can explain all the variation that is not due to likely measurement and within-subject errors. The maximum $r^{2}$ obtainable in a regression analysis is determined by the product of the reliabilities of the $x$ and $y$ variables [6]; a simulation using a synthetic raw data set derived from Table 2 of Gastaldelli et al. [4], and incorporating commonly reported measurement errors for glucose $(3 \% \mathrm{CV})$, insulin (8\%) and clampderived insulin sensitivity $(10 \%)$ produces estimated reliability coefficients of 0.87 and 0.99 for $y$ and $x$ variables respectively, which predict a maximum $r^{2}=0.86$ (compared with $r^{2}=0.83$ [4]). This simulation ignored other potential sources of error, including within-individual errors. The model is therefore sufficient (or saturated) in the sense that there is no room for improvement in the test data. Whether it is sufficient in different data sets is not known at present, 
but could be readily tested in the data of Mari et al. [1]. It cannot be rigorously tested in the data of DeFronzo et al. [2], which include a surrogate index of $S_{\mathrm{i}}$ with only moderate correlation $(r=0.73)$ with measured $S_{\mathrm{i}}$ [7]. If it is generally sufficient, it represents in one physiologically plausible form all the information concerning relationships between $S_{\mathrm{i}}, \beta$ and glycaemia that can be extracted from data of this type. No other model could do better: more complex models would not give reliable estimates of all parameters, and a simpler model is difficult to conceive.

One key feature of this model in the present context is that it provides a simple explanation for the widely accepted hyperbolic relationship between measures of insulin secretion and insulin sensitivity, and that it does so without postulating any mechanistic relationship between $\beta$ and $S_{\mathrm{i}}$. Neither beta cell adaptation to insulin resistance, nor insulin-induced insulin resistance are present in the model. Equation 1 predicts a family of hyperbolic relationships between $\beta$ and $S_{\mathrm{i}}$, one for each level of $G$. In data sets where $G$ varies over a narrow range (e.g. a population sample with normal glucose tolerance), $G$ may contribute comparatively little variance to the right-hand side of Eq. 1, and the result of plotting $\beta$ against $S_{\mathrm{i}}$ may appear to be scatter around a single hyperbola. In data sets with more variation in $G$, the result may be considerable scatter around a hyperbolic function, due to the presence of a family of relationships, which may result in a formally insignificant measure of fit to a single hyperbola. We suggest that this is the reason for the lack of fit to a hyperbola shown in Fig. 1c of Mari et al. [1].

Another key feature of the model relates to the method chosen to express beta cell function, i.e. as glucose sensitivity of insulin secretion. As Mari et al. show [1], the particular measure of insulin secretion used makes a difference to the form of relationship between measured insulin secretion and insulin sensitivity. Their carefully derived measure of glucose sensitivity obtained from OGTT responses showed no significant relationship with $S_{\mathrm{i}}$ for the reasons discussed above, whereas the measure of total insulin output after an i.v. glucose bolus did show a significant hyperbolic fit. If, as seems likely, the bloodglucose response to an i.v. glucose bolus $\left(G_{\text {iv }}\right)$ correlates with the OGTT response $(G)$, an explanation for the discrepancy is apparent. The total insulin output $\left(I_{\mathrm{T}}\right)$, which is a function of the glycaemic response, is related to the glucose sensitivity of insulin secretion multiplied by a correlate of $G$, or:

$\ln \left(I_{\mathrm{T}}\right)=\ln (\beta)+\ln \left(G_{\text {iv }}\right)$

which, by substituting for $\ln (\beta)$ (Eq. 1), becomes:

$\ln \left(I_{\mathrm{T}}\right)=\ln \left(R_{\text {in }}\right)-\ln \left(S_{\mathrm{i}}\right)-\ln (G)+\ln \left(G_{\text {iv }}\right)$
Thus, the influence of $G$ is reduced in Eq. 2 compared with Eq. 1, which will reduce the scatter around the hyperbolic fit of $I_{\mathrm{T}}$ to $S_{\mathrm{i}}$. So the discrepancy shown between Fig. $1 \mathrm{~b}$ and Fig. 1c in Mari et al. [1] is not, in our interpretation, due to different components of insulin secretion, dependent and independent of $S_{\mathrm{i}}$, but rather to two different methods of measuring insulin secretory function. None of this affects the central conclusion of Mari et al., which is that variations in $\beta$ are the major determinant of variations in glucose tolerance.

In contrast to Mari et al, DeFronzo et al. [2] conclude from a similar study that the major determinant of variations in glucose tolerance is variation in the ability of insulin secretion to compensate for the degree of insulin resistance. This conclusion is supported by analysis of an index of insulin secretion, which is one of a number of variants of a disposition index. Disposition indices are defined as the product of a measure of insulin secretion and a measure of insulin sensitivity, which in terms of our model can be expressed as:

$\ln (\mathrm{DI})=\ln (\beta)+\ln \left(S_{\mathrm{i}}\right)$

Substituting for $\ln (\beta)$ and $\ln \left(S_{\mathrm{i}}\right)$ in Eq. 1 results in:

$\ln (G)=\ln \left(R_{\text {in }}\right)-\ln (\mathrm{DI})$

In an OGTT, $R_{\text {in }}$ represents the sum of glucose entry from the gut and endogenous glucose output averaged over the duration of the test, which would not be predicted to vary markedly, and does not appear to be an important omission from the model when applied to the data of Gastaldelli et al. [4]. Therefore, within the model a DI is a function of $1 / G$ plus measurement error (Eq. 4), and conveys no additional information. DeFronzo et al. also report various uncorrected indices of the glucose sensitivity of insulin secretion (variants of $\beta$ in our model) which all appear to show a similar pattern of responses across the groups, and in relation to glycaemia, as do the DIs.

DeFronzo et al. [2] chose to focus on their DI because of the widely accepted theory that there is mechanistic link between insulin secretion and insulin sensitivity, such that decreased insulin sensitivity stimulates increased insulin secretion to maintain glycaemia. This theory was originally developed to explain the hyperbolic relationship between insulin secretion and insulin sensitivity, which within our model is an intrinsic property resulting from the underlying physiology requiring no additional mechanistic explanation. This is not the place for a detailed critique of the DI concept, but one of its implications is relevant to the interpretation by DeFronzo et al. of their results. If a treatment causes an increase in insulin sensitivity $\left(S_{\mathrm{i}}\right)$, but no change in glucose sensitivity of insulin secretion $(\beta)$, this must result in an increased DI (Eq. 3). It may be relevant, therefore, that the data reported by DeFronzo et al. are the baseline for a trial of 
an established insulin-sensitising agent. It can be confidently predicted that: (1) the drug will induce an increase in $S_{\mathrm{i}}$ and therefore DI (unless $\beta$ deteriorates); (2) this increased DI will be interpreted as improved beta cell function even if $\beta$ is not affected; and (3) if $\beta$ does deteriorate this will be offset and obscured in the DI by increased $S_{\mathrm{i}}$, permitting the interpretation that the apparent deterioration of $\beta$ is a benign consequence of the assumed feedback link between $\beta$ and $S_{\mathrm{i}}$. These appear to be highly desirable outcomes for an insulin-sensitising agent in a clinical climate of increasing recognition of the need to treat insulin secretory failure in diabetic and prediabetic states.

Both Mari et al. and DeFronzo et al. support explicitly or implicitly the conclusion that reduced glucose sensitivity of insulin secretion is the most important determinant of impaired glucose tolerance below the diabetic range. Whether this reflects a reduced sensitivity of secretory mechanisms to glucose, a reduced number of functional beta cells as is found in established type 2 diabetes [8], or a combination of the two, remains uncertain.

Duality of interest The authors declare that there is no duality of interest associated with this manuscript.

\section{References}

1. Mari A, Tura A, Natali A et al (2010) Impaired beta cell glucose sensitivity rather than inadequate compensation for insulin resistance is the dominant defect in glucose intolerance. Diabetologia 53:749-756

2. DeFronzo RA, Banerji MA, Bray GA et al (2010) Determinants of glucose tolerance in impaired glucose tolerance at baseline in the Actos Now for Prevention of Diabetes (ACT NOW) Study. Diabetologia 53:435-445

3. Jenkins AB, Furler SM, Campbell LV (2004) Re: Gastaldelli A, Ferrannini E, Miyazaki Y, Matsuda M, DeFronzo RA (2004) Beta cell dysfunction and glucose intolerance: results from the San Antonio metabolism (SAM) study. Diabetologia 47: 31-39. Diabetologia 47:1642-1643

4. Gastaldelli A, Ferrannini E, Miyazaki Y, Matsuda M, DeFronzo RA (2004) Beta-cell dysfunction and glucose intolerance: results from the San Antonio Metabolism (SAM) Study. Diabetologia 47:31-39

5. Matthews DR, Hosker JP, Rudenski AS, Naylor BA, Treacher DF, Turner RC (1985) Homeostasis model assessment: insulin resistance and beta-cell function from fasting plasma glucose and insulin concentrations in man. Diabetologia 28:412-419

6. Lachin JM (2004) The role of measurement reliability in clinical trials. Clin Trials 1:553-566

7. Matsuda M, DeFronzo RA (1999) Insulin sensitivity indices obtained from oral glucose tolerance testing: comparison with the euglycemic insulin clamp. Diab Care 22:1462-1470

8. Wajchenburg BL (2007) $\beta$-Cell failure in diabetes and preservation by clinical treatment. Endocr Rev 28:187-218 